\title{
Effect of plasmonic mode on plasmon-based lasers
}

\author{
Montacer Dridi, ${ }^{1, *}$ Florent Colas, ${ }^{1}$ and Chantal Compère ${ }^{2}$ \\ ${ }^{1}$ Ifremer, REM/RDT/LDCM, F-29280 Plouzané, France \\ ${ }^{2}$ Ifremer, REM/RDT, F-29280 Plouzané, France \\ *Corresponding author: dmontacer@gmail.com
}

Received 3 September 2018; revised 4 November 2018; accepted 5 November 2018; posted 5 November 2018 (Doc. ID 344749); published 29 November 2018

\begin{abstract}
For the last few years, plasmon-based lasers have been investigated theoretically and experimentally. Several configurations have been reported. They are composed of quantum emitters coupled to a plasmonic structure. In this paper, we investigate the effect of the plasmon mode on the far-field and near-field characteristics of a nanolaser composed by a periodic array of metallic nanoparticles covered by gain materials under optical pumping. Two configurations were investigated. The first structure supported a localized surface plasmon (LSP) mode, while the second a surface lattice plasmon (SLP) mode. This theoretical work relies on semi-quantum calculations based on a four-level gain molecule and a time-domain approach. We demonstrated that lasing in a SLP mode requires a lower concentration of quantum emitters and generates a higher far-field emission with a lower threshold than lasing in a LSP mode. At nanoscale, near-field enhancement at the emission wavelength and above the threshold is 2 orders of magnitude higher with the SLP than the LSP mode. Our results with a general character show that designing a plasmon-based laser that sustains the SLP mode will result in a better lasing efficiency than with a LSP mode. (c) 2018 Optical Society of America
\end{abstract}

https://doi.org/10.1364/JOSAB.35.003110

\section{INTRODUCTION}

Periodic arrays of metallic nanoparticles have remarkable optical properties associated with the excitation of surface plasmons, making them of great interest in sensing [1-12] and lasing $[6,13,14,15,16,17,18]$. When illuminated by a plane wave, dipolar and higher order plasmonic modes could be excited as well as the surface lattice plasmon (SLP). The latter is the result of coupling diffraction orders to a localized surface plasmon (LSP) mode. The SLP exhibits significant improved properties when compared to LSP resonance [19-24]. Improvements include high-quality peak [25-27], angle-dependent dispersion [28], sensitivity to refractive index [29], and higher near-field enhancement $[30,31]$. The SLP has been successfully used for lasing in Refs. [32-37]. In Ref. [32], the authors reported lasing by band edge lattice plasmons in arrays of gold nanocavities in a homogeneous dielectric environment doped with IR-140 dye. Several plasmon-based lasers have been theoretically proposed and experimentally demonstrated using periodic plasmonic cavities sustaining electromagnetic resonance that does not satisfy the SLP condition [38,39]. More specifically in Ref. [40], it was demonstrated theoretically that the type of lasing mode can be tuned by modifying the grating constant. In this paper, we expose the first systematic comparison between lasing in SLPs and lasing in the LSP mode. To this end, we investigate theoretically two different plasmon-based lasers composed of an array of gold nanorods. The first array has a period $p=300 \mathrm{~nm}$ and supports LSP mode, while the second has a period $p=525 \mathrm{~nm}$ and sustains a SLP mode. Both arrays are covered by an active layer containing a fluorophore as a quantum emitter at a concentration $C_{0}=40 \mu \mathrm{mol} \cdot \mathrm{mL}^{-1}$.

The paper is structured as follow: in the first section, the theory and modeling used to describe gain molecules interacting with plasmonic field are reviewed. In the second section, the optical properties of the so-called passive structure, which corresponds to a concentration of the gain molecule equal to 0 , are investigated. The third section exposes the far-field properties of lasing: emission intensity, mechanism of lasing, and dependence to the concentration of gain media. In the fourth section, the near fields associated with lasing in each mode are compared.

\section{BRIEF REVIEW OF THE THEORETICAL APPROACH}

In order to model interactions between the gain molecules and the plasmonic resonator, a semi-quantum approach was developed in Refs. $[32,39,40]$. Briefly, in such an approach, the electromagnetic field is treated classically by solving the time domain Maxwell equations,

$$
\nabla \times \vec{E}(t)=-\mu_{0} \frac{\partial \vec{H}(t)}{\partial t},
$$




$$
\nabla \times \vec{H}(t)=\epsilon \frac{\partial \vec{E}(t)}{\partial t}+\frac{\partial \vec{P}(t)}{\partial t},
$$

where in Faraday's law, we introduce a current $\vec{J}=\frac{\partial \vec{P}}{\partial t}$ to account for the modification of the total field due to the presence of the quantum emitters. $\vec{P}$ is the net macroscopic polarization of the gain material. The quantum emitters are described as four level molecules (Fig. 1). $\vec{P}$ is the consequence of the absorption transition from state 0 to state 3 and the emission transition from state 2 to 1 . Other transitions are assumed to be very fast and negligible. $\vec{P}_{21}$ and $\vec{P}_{03}$ are both calculated by solving [41-44]

$$
\frac{d^{2} \vec{P}_{i, j}(t)}{d t^{2}}+\Delta \omega_{i, j} \frac{d \vec{P}_{i, j}}{d t}+\omega_{i, j}^{2} \vec{P}_{i, j}=\kappa_{i, j} \rho_{i, j}(t) \vec{E}(t) .
$$

$\Delta \omega_{i, j}$ is the bandwidth of the transition of interest, and $\kappa_{i, j}=$ $6 \pi \epsilon_{0} c^{3} / \omega_{i, j}^{2} \gamma_{i, j}$ and $\rho_{i, j}=\rho_{j}-\rho_{i}$ are the population density difference between the upper and lower states. $\gamma_{i, j}$ is the spontaneous decay rate.

The population density of the fundamental and excited states $\rho_{i, j}(t)$ could be derived from the rates equations approach,

$$
\begin{gathered}
\dot{\rho}_{3}=-\frac{\rho_{3}(t)}{\tau_{32}}-\frac{\rho_{3}(t)}{\tau_{30}}+\frac{1}{\hbar \omega_{30}} \vec{E}(t) \cdot \frac{d \vec{P}_{30}(t)}{d t}, \\
\dot{\rho}_{2}=\frac{\rho_{3}(t)}{\tau_{32}}-\frac{\rho_{2}(t)}{\tau_{21}}+\frac{1}{\hbar \omega_{21}} \vec{E}(t) \cdot \frac{d \vec{P}_{21}(t)}{d t}, \\
\dot{\rho}_{1}=\frac{\rho_{2}(t)}{\tau_{21}}-\frac{\rho_{1}(t)}{\tau_{10}}-\frac{1}{\hbar \omega_{21}} \vec{E}(t) \cdot \frac{d \vec{P}_{21}(t)}{d t}, \\
\dot{\rho}_{0}=\frac{\rho_{1}(t)}{\tau_{10}}+\frac{\rho_{3}(t)}{\tau_{30}}-\frac{1}{\hbar \omega_{30}} \vec{E}(t) \cdot \frac{d \vec{P}_{30}(t)}{d t} .
\end{gathered}
$$

The above set of nonlinear coupled equations describes the whole spatiotemporal dynamics of a four-level energy system interacting with electromagnetic field including metallic nanoparticles. The equations are solved in a three-dimensional scheme, according to the technique described in Ref. [39].

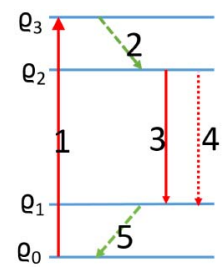

Fig. 1. Transitions accounted in the gain molecule: 1 , Absorption transition with a pumping rate $W_{p}=\frac{1}{h \omega_{30}} \vec{E} \cdot \frac{d \vec{P}_{30}}{d t} .2$, Very fast spontaneous transition with decay time $\tau_{32}=10 \mathrm{fs}$. 3, Stimulated transition with an emission rate $W_{e}=\frac{1}{h \omega_{21}} \vec{E} \cdot \frac{d \vec{P}_{30}}{d t}$. 4, Spontaneous emission with slow decay time $\tau_{21}(x, y, z)=\tau_{21}^{0} / P f_{x, y, z}$ with $\tau_{21}^{0}=1 \mathrm{~ns}$, and $P f_{x, y, z}$ is the Purcell factor calculated in Ref. [39]. 5, Very fast spontaneous transition with decay time $\tau_{10}=10 \mathrm{fs}$.

\section{OPTICAL PROPERTIES OF THE PASSIVE STRUCTURE}

Let us first describe the optical response of the passive structure (without the fluorophore). Two different samples are considered in this study. They consisted in 2D arrays of gold nanorods embedded in an infinite lossless dielectric host material, whose refractive index equals 1.5 . The nanorods are $100 \mathrm{~nm}$ long, $50 \mathrm{~nm}$ wide, and $60 \mathrm{~nm}$ high. The two samples differ in the period $p$ of the grating: $300 \mathrm{~nm}$ and $525 \mathrm{~nm}$. A light beam impinges the sample orthogonally to the grating plan. The polarization is along the long axis of the nanorods (Fig. 2). Figure 3 summarizes the spectral response of the passive nanostructures. Figure 3(a) shows the extinction spectra for the array with $p=300 \mathrm{~nm}$. It shows a resonance mode at $721 \mathrm{~nm}$ corresponding to a LSP mode, characterized by a very weak near-field coupling between the proximal nanoparticle (the near-field extension of an individual nanoparticle is $\ll p$ ) and an absence of a matching with a diffraction order. Figure $3(\mathrm{~b})$ corresponding to $p=525 \mathrm{~nm}$

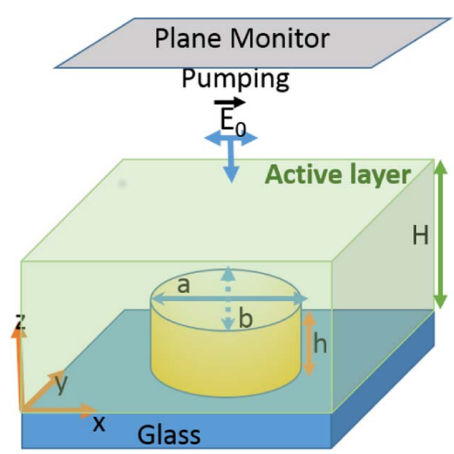

Fig. 2. Description of the unit cell in the plasmon-based laser.
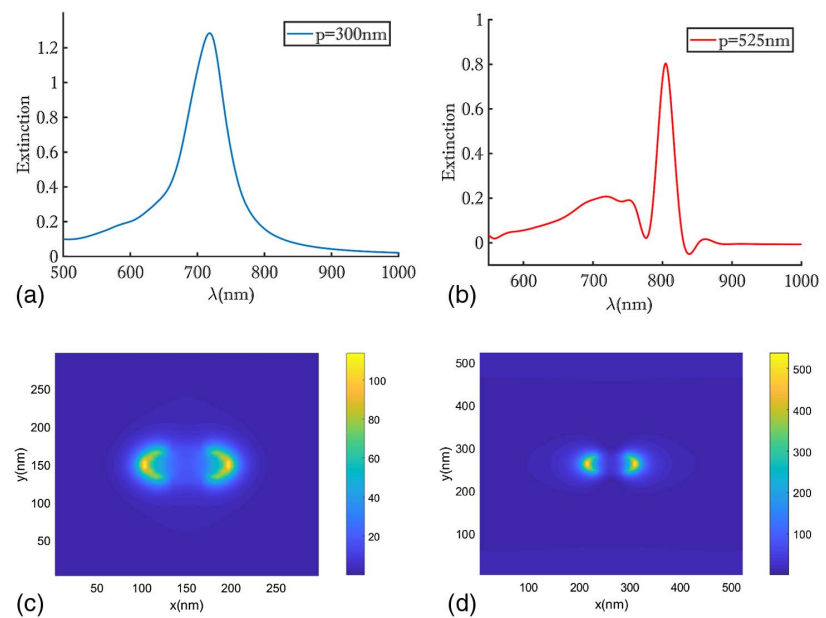

Fig. 3. Optical properties of the passive structures. (a) Calculated extinction spectra for $p=300 \mathrm{~nm}$. A localized plasmon resonance mode is located at $\lambda=721 \mathrm{~nm}$. (b) Calculated extinction spectra for $p=525 \mathrm{~nm}$. A surface plasmon resonance mode is located at $\lambda=810 \mathrm{~nm}$, indicated by a narrower peak and a broader resonance at $\lambda=721 \mathrm{~nm}$. (c), (d) Calculated near-field enhancement (c) at $\lambda=$ $721 \mathrm{~nm}$ with $p=300 \mathrm{~nm}$ and (d) at $\lambda=810 \mathrm{~nm}$ with $p=525 \mathrm{~nm}$ in a plane parallel to the substrate at $10 \mathrm{~nm}$ from the nanorod top surface. 
shows spectra with two resonance modes. The lower energy mode located at $\lambda=810 \mathrm{~nm}$ features weak nonradiative losses (narrower spectra) due to the strong coupling between a diffracted wave and a plasmonic mode. The energy in that case is captured in the form of electronic oscillations (plasmon) rather than photons diffracted in the far field. It corresponds to a SLP mode. The higher energy mode, located at $\lambda=720 \mathrm{~nm}$, shows a poor quality factor and corresponds roughly to the the LSP mode of an isolated nanoparticle. Figures 3(c) and 3(d) show the near-field enhancement $\left(\left|\frac{\vec{E}}{\vec{E}_{0}}\right|^{2}\right)$ map at the resonance wavelength. As predicted previously $[19,45]$, the enhancement field in the case of the SLP mode is stronger than in the case of the LSP mode.

\section{FAR-FIELD CHARACTERISTIC OF THE LASING}

Now, the nanoarray is embedded in a dielectric host containing a gain molecule with emission properties detailed below. The thickness of the active layer is fixed to $200 \mathrm{~nm}$. We carried out a series of numerical experiments in which the emission wavelength of the gain molecule is set to $\lambda_{e}=720 \mathrm{~nm}$ and the absorption wavelength $\lambda_{a}=600 \mathrm{~nm}$. Each of those transitions has a spectral bandwidth $\Delta \lambda_{e}=\Delta \lambda_{e}=100 \mathrm{~nm}$. The decay rates are depicted in the legend of Fig. 1 . We also chose a reasonable value of the volumic concentration of the gain molecule $C_{0}=25 * 10^{24}$ molecule $\cdot \mathrm{m}^{-3} \approx 40 \mu \mathrm{mol} \cdot \mathrm{mL}^{-1}$.

In order to elucidate the feedback mechanism in the lasing process, emission spectra were calculated for two different distributions of the gain molecule in the active layer. The first distribution denoted $D$ corresponds to a strictly homogeneous spatial distribution of the molecules. In the second distribution (noted $D^{\prime}$ ), we assume that the closest molecule to the nanoparticle (NP) is situated at a distance $d=25 \mathrm{~nm}$. There is no gain molecule at a distance lower than $25 \mathrm{~nm}$. By doing so, we can investigate the contribution of gain molecules that are very close to the NP to the lasing emission. Finally, to allow a quantitative comparison between lasing intensity in different geometries and concentrations, the emission intensity is normalized by the emission obtained for the array with $p=300 \mathrm{~nm}$ and $C_{0}$ at the maximum pump fluence used $\left(3.6 \mathrm{~mJ} \cdot \mathrm{cm}^{-2}\right)$. This normalization is the same normalization used through the paper. Figure 4 summarizes the far-field emission observed for each array as the gain molecules are assumed initially to be at the ground state. Figures 4(a) and 4(b) present the calculated emission spectra above the threshold for $p=300 \mathrm{~nm}$ and $p=525 \mathrm{~nm}$, respectively. The former shows a lasing peak at $\lambda=732 \mathrm{~nm}$ and the latter at $\lambda=810 \mathrm{~nm}$, which correspond to the LSP and the SLP mode, respectively.

The existence of a laser threshold is presented in Figs. 4(c) and 4(d), where we plot the calculated emission intensity as a function of the pumping energy in each case of the distributions of gain molecules $D$ and $D^{\prime}$. Several points of information come out from the plot: first, the emission intensity when lasing in the SLP mode is 2 orders of magnitude higher than the lasing in LSP mode. Second, despite a greater overlap between the emission of the gain molecule spectrum $\left(\lambda_{e}=720 \mathrm{~nm}\right)$ with the LSP mode than the SLP mode, the threshold of lasing
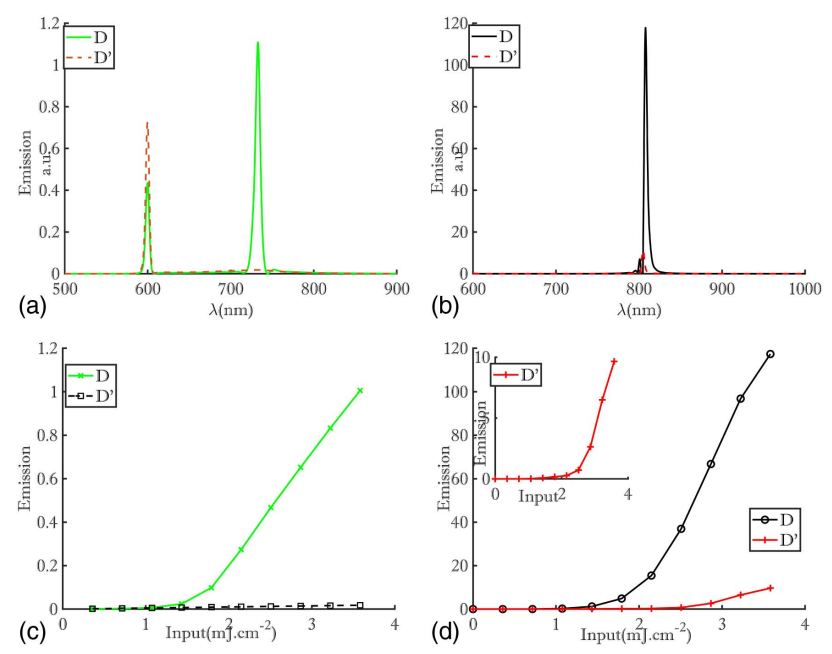

Fig. 4. (a) Emission spectra for a laser that has a lattice spacing of $300 \mathrm{~nm}$. A peak at $\lambda=732 \mathrm{~nm}$ associated with LSP mode is observed, with a narrow bandwidth $(\Delta \lambda=8 \mathrm{~nm})$. (b) Emission spectra for a laser that has a lattice spacing of $525 \mathrm{~nm}$. A peak at $\lambda=810 \mathrm{~nm}$ associated with the LSP mode is observed with a very narrow bandwidth $\Delta \lambda=4 \mathrm{~nm}$. (c) Emission calculated at the lasing peak for $p=$ $300 \mathrm{~nm}$ as a function of pumping energy. Emission shows a clear threshold when gain molecules are located close to the NP. The black dashed line represents the emission when gain molecules are located at $25 \mathrm{~nm}$ away from the NP surface $\left(D^{\prime}\right)$, making lasing impossible. (d) Threshold dependence at $\lambda=810 \mathrm{~nm}(p=525 \mathrm{~nm})$. Inset, calculated emission with $D^{\prime}$ showing the threshold characteristic with weak emission intensity compared to $D$.

is approximately the same. It suggests then that the threshold for lasing in SLP mode is lower than lasing in LSP mode. Third, the lasing in the LSP and SLP relies on different modes for feedback, although the near-field distributions in the both passive structures are similar (Fig. 2). Indeed, when there is no gain molecule at the very close vicinity of the gold surface $\left(D^{\prime}\right.$ as the distribution of gain molecule), the emission was impossible in the case of the LSP mode but could be obtained in the case of the SLP mode. The emission intensity in the case of distribution D' and SLP mode is an order of magnitude higher than in the case of distribution D and a LSP mode.

In the last part of this section, the emission for different concentrations of the gain material is investigated. We limit our study to the pumping fluence comprised between 0 and $3.6 \mathrm{~mJ} \cdot \mathrm{cm}^{-2}$. Figure $5(\mathrm{a})$ represents a plot of the emission as a function of the input for different concentrations ranging from $\left[\frac{C_{0}}{5}-C_{0}\right]$ for a period $p=300 \mathrm{~nm}$ (system lasing in LSP mode), and since we observe that the lasing effect is impossible for a concentration less than $\frac{C_{0}}{2}$, the increase of $C$ up to $2 C_{0}$ leads to a significant increase of the threshold of lasing without any significant increase of the emission in the far field. This is attributed to the high absorption of the active layer for high concentration of the gain molecule, which reduces significantly the gain $\left(\rho_{2}-\rho_{1}\right)$ close to the NP. Indeed, the incident field that propagates through the active layer is attenuated before reaching the NP. For a system lasing in SLP mode $(p=525 \mathrm{~nm})$, a dramatic different behavior is observed. Indeed in Fig. 5(c), the lasing effect is possible for a very wide 

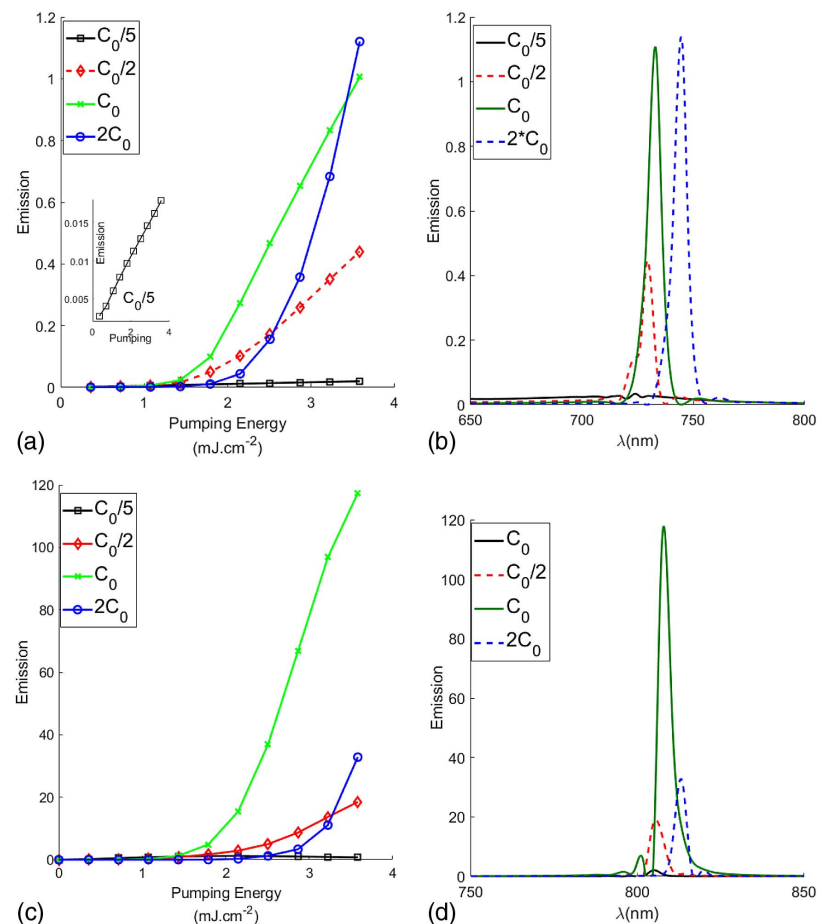

Fig. 5. Dependence of the far-field lasing emission on the concentration of the gain molecule. (a) Lasing in the LSP mode. Inset, emission with $C_{0} / 5$ showing that lasing is impossible. (b) Shift of the LSP lasing wavelength for different concentrations. (c) Lasing in the SLP mode for different concentrations. (d) Shift of the SLP lasing wavelength for different concentrations.

range of concentration, even for very weak concentration $\left(\frac{C_{0}}{5}\right)$. However, the emission intensity in the far field is strongly dependent on $C$. For example, the emission intensity increases from 1 to 120 when the concentration goes from $\frac{C_{0}}{5}$ to $C_{0}$. Note that the emission intensity in the case of the SLP mode using $\frac{C_{0}}{5}$ is equal to the emission intensity in the LSP mode using the concentration $C_{0}$.

We explain our results by the fact that the lasing mechanism in the LSP and SLP modes are dramatically different from each other. Lasing in the LSP mode relies only on the plasmonic localized mode for feedback, and thus the gain molecules contributing to the lasing are localized in a very small volume close to the NP. In that case, a high density of molecules in this volume is required. On the other hand, lasing in the SLP mode relies on the geometry (periodic arrangement) and on the plasmonic mode. Thus, all the molecules in the active layer contribute to the lasing, and energy transfer here is mediated by in-plane diffracted waves.

In Figs. 5(b) and 5(d), we calculate the emission above the threshold for different $C$, and we observe that the laser wavelength is redshifted when increasing the concentration $C$. We attribute this redshift to the increase of the effective refractive index in the active layer with the fluorophore concentration.

To conclude this section, we have demonstrated that using a high concentration of gain molecules, lasing in the SLP mode will generate an emission intensity in the far field that is 2 orders of magnitude higher than lasing in the LSP mode.
We demonstrated also that an increase of the concentration of the fluorophore may result in an increase of the lasing threshold and a redshift of the lasing emission wavelength. Finally, we demonstrated that lasing in the LSP mode using a weak concentration of gain (less than $10 \mu \mathrm{mol} \cdot \mathrm{mL}^{-1}$ ) is impossible.

\section{NEAR-FIELD CHARACTERISTICS}

In this section, we compare the near-field intensity in the two arrays of nanoparticles at a pump fluence above the threshold. For this goal, we calculate $\left|E / E_{0}\right|^{2}$ with $E$, the total field calculated in the active system, and $E_{0}$ is the incident field. For the two arrays of nanoparticles, $E_{0}$ is a pump pulse with a temporal bandwidth equal to $150 \mathrm{fs}$ and centered at the absorption wavelength of the gain molecule $(600 \mathrm{~nm})$. In Figs. 6(a) and 6(b), we plot the enhancement $\left(\left|E / E_{0}\right|^{2}\right)$ spectra calculated close to the NP for an intensity of a pumping intensity above the threshold (equal to $3.6 \mathrm{~mJ} \cdot \mathrm{cm}^{-2}$ ). We observe that in each case, the near field in the active structure presents a resonant wavelength that is very close to the emission wavelength in the far field. The emission of photons observed in the far field is associated at nanoscale with an intense localized near field.

When comparing Figs. 6(a) and 6(b), we note that the enhancement is 4 orders of magnitude higher in the case of the SLP mode than the LSP modes, showing again that lasing in the SLP mode has better quality and characteristics. In Figs. 6(c) and 6(b), we compare the field distribution above the threshold for the LSP and SLP modes. For the LSP mode, the field is more localized around the NP edge. In the SLP mode case, the near field extends far to the NP edge. An enhancement of $10^{7}$ is observed very close to the NP. A significant enhancement of $10^{5}$ located $200 \mathrm{~nm}$ away from the NP edge is observed. Also for each structure, the enhancement achieved is several orders of magnitude higher than the corresponding passive structure.

To conclude this section, we have made the first demonstration here, to the best of our knowledge, that the local electric fields within the active nanostructure can achieve strengths that
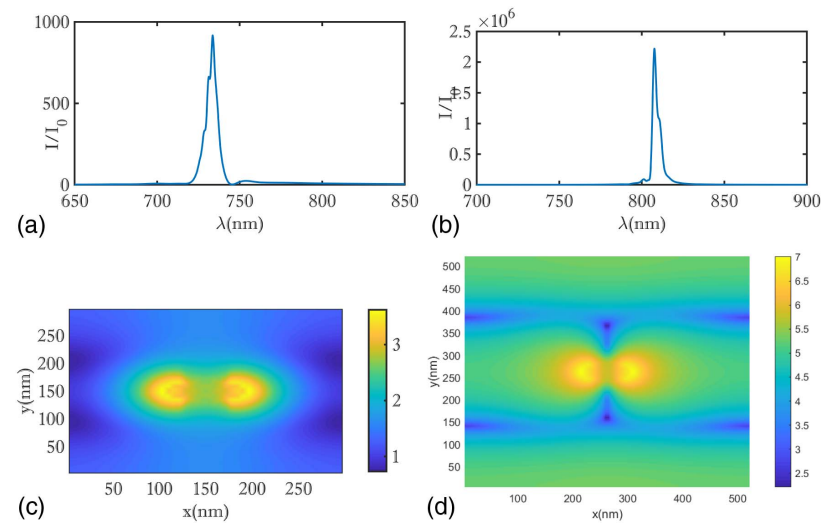

Fig. 6. Near-field properties associated with lasing. (a) Enhancement spectra averaged around the nanoparticle calculated above the threshold. Maximum enhancement is observed at (a) the LSP mode and (b) the SLP mode. (c) Map of the field above the threshold at the LSP wavelength. (d) Map of the field above the threshold at the SLP wavelength. 
are orders of magnitude higher than those of the corresponding passive structure. The enhancement achieved when lasing in SLP mode is 3-4 orders of magnitude higher than lasing in LSP mode. Moreover, the near-field enhancement at the SLP mode extends even away from the nanoparticle, which could be extremely useful for sensing applications as the sensing volume increases significantly.

More generally, we have made here the first demonstration, to the best of our knowledge, of the near-field enhancement associated with lasing emission in an active structure, suggesting that hybridizing plasmonic nanoparticles with molecules exhibiting gain could be a very powerful tool to generate an intense near field at nanoscale. This will be of a great interest for enhancing a weak Raman signal and developing a novel class of sensors.

\section{CONCLUSION}

We have presented in this paper, to the best of our knowledge, the first quantitative and qualitative comparison of lasing action in LSP and SLP modes in an array of gold nanorods covered by a dielectric material doped with gain molecules. Our results reveal that lasing in the SLP mode presents three distinct advantages: a higher emission intensity in the far field associated with an intense near-field enhancement, a lower threshold, and lower concentration of gain molecule requirement. However, the design and fabrication of a plasmon-based laser when using SLP resonances are more challenging due to the necessary relationship between the diffractive order and LSP resonance wavelength. More generally, our results demonstrate the potential of the plasmon laser for the generation of ultraintense local field at nanoscale. They could be of great importance for further development of novel light-emitting structures based on active plasmonic crystals.

Funding. Conseil Régional de Bretagne; Institut Français de Recherche pour l'Exploitation de la Mer (IFREMER), Direction Scientifique.

\section{REFERENCES}

1. B. Špačková and J. Homola, "Sensing properties of lattice resonances of 2D metal nanoparticle arrays: an analytical model," Opt. Express 21, 27490-27502 (2013).

2. D. A. Stuart, A. J. Haes, A. D. McFarland, S. Nie, and R. P. V. Duyne, "Refractive-index-sensitive, plasmon-resonant-scattering, and surfaceenhanced Raman-scattering nanoparticles and arrays as biological sensing platforms," Proc. SPIE 5327, 60-74 (2004).

3. C. Valsecchi and A. G. Brolo, "Periodic metallic nanostructures as plasmonic chemical sensors," Langmuir 29, 5638-5649 (2013).

4. J. Aizpurua, P. Hanarp, D. S. Sutherland, M. Käll, G. W. Bryant, and F. J. García de Abajo, "Optical properties of gold nanorings," Phys. Rev. Lett. 90, 057401 (2003).

5. Y. Shen, J. Zhou, T. Liu, Y. Tao, R. Jiang, M. Liu, G. Xiao, J. Zhu, Z.-K. Zhou, X. Wang, C. Jin, and J. Wang, "Plasmonic gold mushroom arrays with refractive index sensing figures of merit approaching the theoretical limit," Nat. Commun. 4, 2381 (2013).

6. Y.-F. C. Chau, C.-K. Wang, L. Shen, C. M. Lim, H.-P. Chiang, C.-T. C. Chao, H. J. Huang, C.-T. Lin, N. T. R. N. Kumara, and N. Y. Voo, "Simultaneous realization of high sensing sensitivity and tunability in plasmonic nanostructures arrays," Sci. Rep. 7, 16817 (2017).

7. J. D. Caldwell, O. J. Glembocki, F. J. Bezares, M. I. Kariniemi, J. T. Niinistö, T. T. Hatanpää, R. W. Rendell, M. Ukaegbu, M. K. Ritala,
S. M. Prokes, C. M. Hosten, M. A. Leskelä, and R. Kasica, "Large-area plasmonic hot-spot arrays: sub-2 $\mathrm{nm}$ interparticle separations with plasma-enhanced atomic layer deposition of $\mathrm{Ag}$ on periodic arrays of Si nanopillars," Opt. Express 19, 26056-26064 (2011).

8. K.-L. Lee, C.-H. Cheng, W.-Y. Chang, and P.-K. Wei, "Dual SPRSERS sensors using gold nanoslits and oblique angle deposition," in JSAP-OSA Joint Symposia 2014 Abstracts (Optical Society of America, 2014), paper 18a_C4_5.

9. S. Wei, M. Zheng, Q. Xiang, H. Hu, and H. Duan, "Optimization of the particle density to maximize the SERS enhancement factor of periodic plasmonic nanostructure array," Opt. Express 24, 20613-20620 (2016).

10. J. Olavarría-Fullerton, R. A. Velez, S. Wells, M. J. Sepaniak, S. P. Hernández-Rivera, and M. A. D. Jesús, "Design and characterization of hybrid morphology nanoarrays as plasmonic Raman probes for antimicrobial detection," Appl. Spectrosc. 67, 1315-1322 (2013).

11. P. Mandal and S. A. Ramakrishna, "Dependence of surface enhanced Raman scattering on the plasmonic template periodicity," Opt. Lett. 36, 3705-3707 (2011).

12. T. Siegfried, Y. Ekinci, H. Solak, and O. Martin, "Large area periodic nanogap arrays for Raman and fluorescence enhancement: modeling and performance," in Conference on Lasers and Electro-Optics (Optical Society of America, 2012), paper QM3H.3.

13. M. I. Stockman, "Nanoplasmonics: past, present, and glimpse into future," Opt. Express 19, 22029-22106 (2011).

14. N. P. N. I. Zheludev, S. L. Prosvirnin, and V. A. Fedotov, "Lasing spaser," Nat. Photonics 2, 351-354 (2008).

15. D. J. Bergman and M. I. Stockman, "Surface plasmon amplification by stimulated emission of radiation: quantum generation of coherent surface plasmons in nanosystems," Phys. Rev. Lett. 90, 027402 (2003).

16. M. Ramezani, A. Halpin, A. I. Fernández-Domínguez, J. Feist, S. R.-K. Rodriguez, F. J. Garcia-Vidal, and J. G. Rivas, "Plasmon-excitonpolariton lasing," Optica 4, 31-37 (2017).

17. H.-Y. Wu, L. Liu, M. Lu, and B. T. Cunningham, "Lasing emission from plasmonic nanodome arrays," in Conference on Lasers and ElectroOptics (Optical Society of America, 2016), paper FTh1B.8.

18. Z. Wang, X. Meng, S. H. Choi, Y. L. Kim, V. M. Shalaev, and A. Boltasseva, "Plasmonic random lasing in strongly scattering regime with slanted silver nanorod array," in Conference on Lasers and Electro-Optics (Optical Society of America, 2015), paper FW3E.7.

19. S. Zou, N. Janel, and G. C. Schatz, "Silver nanoparticle array structures that produce remarkably narrow plasmon lineshapes," J. Chem. Phys. 120, 10871-10875 (2004).

20. Y. Chu, E. Schonbrun, T. Yang, and K. B. Crozier, "Experimental observation of narrow surface plasmon resonances in gold nanoparticles arrays," Appl. Phys. Lett. 93, 181108 (2008).

21. W. Zhou and T. W. Odom, "Tunable subradiant lattice plasmons by out-of-plane dipolar interactions," Nat. Nanotechnol. 6, 423-427 (2011).

22. E. M. Hicks, S. Zou, G. C. Schatz, K. G. Spears, and R. P. Vduyne, "Controlling plasmon line shapes through diffractive coupling in linear arrays of cylindrical nanoparticles fabricated by electron beam lithography," Nano Lett. 5, 1065-1070 (2005).

23. A. G. Nikitin, A. V. Kabashin, and H. Dallaporta, "Plasmonic resonances in diffractive arrays of gold nanoantennas: near and far field effects," Opt. Express 20, 27941-27952 (2012).

24. A. B. Evlyukhin, C. Reinhardt, U. Zywietz, and B. N. Chichkov, "Collective resonances in metal nanoparticle arrays with dipolequadrupole interactions," Phys. Rev. B 85, 245411 (2012).

25. V. G. Kravets, F. Schedin, and A. N. Grigorenko, "Extremely narrow plasmon resonances based on diffraction coupling of localized plasmons in arrays of metallic nanoparticles," Phys. Rev. Lett. 101, 087403 (2008).

26. B. Auguié and W. L. Barnes, "Collective resonances in gold nanoparticle arrays," Phys. Rev. Lett. 101, 143902 (2008).

27. A. D. Humphrey and W. L. Barnes, "Plasmonic surface lattice resonances on arrays of different lattice symmetry," Phys. Rev. B 90, 075404 (2014).

28. D. Khlopin, F. Laux, W. P. Wardley, J. Martin, G. A. Wurtz, J. Plain, N. Bonod, A. V. Zayats, W. Dickson, and D. Gérard, "Lattice modes and plasmonic linewidth engineering in gold and aluminum nanoparticle arrays," J. Opt. Soc. Am. B 34, 691-700 (2017). 
29. A. Danilov, G. Tselikov, F. Wu, V. G. Kravets, I. Ozerov, F. Bedu, A. N. Grigorenko, and A. V. Kabashin, "Ultra-narrow surface lattice resonances in plasmonic metamaterial arrays for biosensing applications," Biosens. Bioelectron. 104, 102-112 (2018).

30. Z. Cao, L. Zhang, C.-Y. Chan, and H.-C. Ong, "Interplay between absorption and radiative decay rates of surface plasmon polaritons for field enhancement in periodic arrays," Opt. Lett. 39, 501-504 (2014).

31. S. R. K. Rodriguez, O. T. A. Janssen, G. Lozano, A. Omari, Z. Hens, and J. G. Rivas, "Near-field resonance at far-field-induced transparency in diffractive arrays of plasmonic nanorods," Opt. Lett. 38, 1238-1240 (2013).

32. W. Zhou, M. Dridi, J. Y. Suh, C. H. Kim, D. T. Co, M. R. Wasielewski, G. C. Schatz, and T. W. Odom, "Lasing action in strongly coupled plasmonic nanocavity arrays," Nat. Nanotechnol. 8, 506-511 (2013).

33. A. Yang, T. B. Hoang, M. Dridi, M. H. M. Claire Deeb, G. C. Schatz, and T. W. Odom, "Real-time tunable lasing from plasmonic nanocavity arrays," Nat. Commun. 6, 6939 (2015).

34. C. Deeb, Z. Guo, A. Yang, L. Huang, and T. W. Odom, "Correlating nanoscopic energy transfer and far-field emission to unravel lasing dynamics in plasmonic nanocavity arrays," Nano Lett. 18, 1454-1459 (2018).

35. J. Y. S. C. H. Kim, W. Zhou, M. D. Huntington, D. T. Co, M. R. Wasielewski, and T. W. Odom, "Plasmonic bowtie nanolaser arrays," Nano Lett. 12, 5769-5774 (2012).

36. A. H. Schokker and A. F. Koenderink, "Lasing in quasi-periodic and aperiodic plasmon lattices," Optica 3, 686-693 (2016).
37. T. K. Hakala, H. T. Rekola, A. I. Väkeväinen, J.-P. Martikainen, M. Nečada, A. J. Moilanen, and P. Törmä, "Lasing in dark and bright modes of a finite-sized plasmonic lattice," Nat. Commun. 8, 13687 (2017).

38. F. van Beijnum, P. J. van Veldhoven, E. J. Geluk, M. J. A. de Dood, G. W. 't Hooft, and M. P. van Exter, "Surface plasmon lasing observed in metal hole arrays," Phys. Rev. Lett. 110, 206802 (2013).

39. M. Dridi and G. C. Schatz, "Model for describing plasmon-enhanced lasers that combines rate equations with finite-difference timedomain," J. Opt. Soc. Am. B 30, 2791-2797 (2013).

40. M. Dridi and G. C. Schatz, "Lasing action in periodic arrays of nanoparticles," J. Opt. Soc. Am. B 32, 818-823 (2015).

41. A. Taflove and S. C. Hagness, Computational Electrodynamics: The Finite-Difference Time-Domain Method (Artech House, 2000).

42. A. E. Siegman, Lasers (University Science Books Mill Valley, 1976).

43. A. S. Nagra and R. A. York, "FDTD analysis of wave propagation in nonlinear absorbing and gain media," IEEE Trans. Antennas Propag. 46, 334-340 (1998).

44. Y. F. P. Bermel, E. Lidorikis, and J. D. Joannopoulos, "Active materials embedded in photonic crystals and coupled to electromagnetic radiation," Phys. Rev. B 73, 165125 (2006).

45. L. Zhao, K. L. Kelly, and G. C. Schatz, "The extinction spectral of silver nanoparticle arrays: influence of array structure on plasmon resonance wavelength and width," J. Phys. Chem. B 107, 7343-7350 (2003). 